\title{
Darf der Beschuldigte im Strafverfahren lügen?
}

Marc Thommen*

Obwohl im Strafverfahren das Schweigen des Beschuldigten de facto als Zustimmung zur Anklage gedeutet wird, tut man sich nach wie vor schwer, ein Recht zur Lüge zu akzeptieren. Die Wahrheit sagen muss er nicht, lügen darf er nicht? Damit hängt der Beschuldigte merkwürdig im luftleeren Raum. Ein ausdrückliches Lügerecht besteht nicht und ein solches lässt sich auch nicht aus dem Nemo-teneturGrundsatz ableiten. Es ergibt sich vielmehr aus seinem Anspruch auf rechtliches Gehör und aus seiner Meinungsäusserungsfreiheit. Das Recht sich im Verfahren zu äussern ist nicht auf wahre Aussagen beschränkt. Der Beschuldigte hat somit das Recht im Strafverfahren zu lügen.

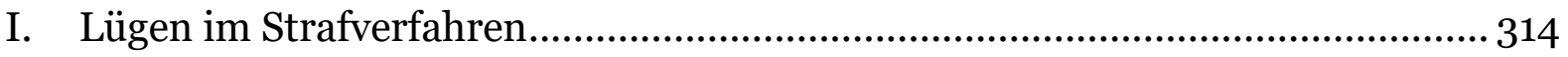

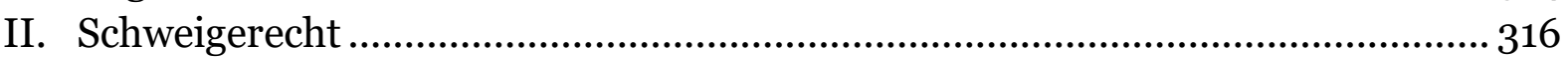

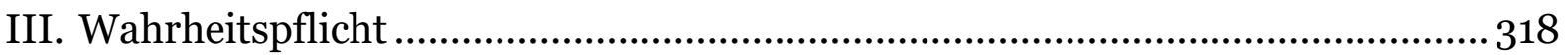

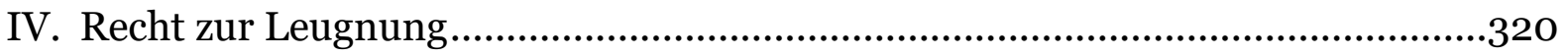

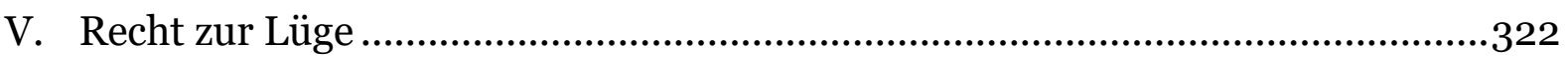

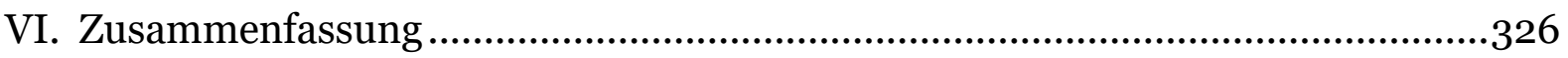

Zitiervorschlag: Marc Thommen, Darf der Beschuldigte im Strafverfahren lügen?, in: sui-generis 2018, S. 313

URL: $\quad$ sui-generis.ch/76

DOI: $\quad$ https://doi.org/10.21257/sg.76

* Marc Thommen (marc.thommen[at]rwi.uzh.ch), Prof. Dr. iur., LL.M. (Cantab), ist Lehrstuhlinhaber für Strafrecht und Strafprozessrecht unter Einschluss des Wirtschafts- und Verwaltungsstrafrechts an der Universität Zürich.

Dieses Werk ist lizenziert unter einer Creative Commons Namensnennung - Weitergabe unter gleichen Bedingungen 4.0 International Lizenz. 


\section{Lügen im Strafverfahren}

1 «Dass Du lügen darfst, heisst nicht unbedingt, dass Du totalen Quatsch erzählen sollst!» Bill Clinton ${ }^{1}$

«I did not have sexual relations with that woman», sagte Bill Clinton am 26. Januar 1998 in einer Fernsehansprache. Damit reagierte der sichtlich zerknirschte 42. Präsident der Vereinigten Staaten von Amerika auf Gerüchte, er habe eine aussereheliche Beziehung zu Monica Lewinsky, einer Praktikantin im Weissen Haus, unterhalten. Solche «sexuellen Beziehungen» mit Monica Lewinsky hatte Bill Clinton bereits zuvor in einem Verfahren wegen sexueller Belästigung von Paula Jones unter Eid bestritten. Bereits die distanzierende Wortwahl, Clinton sprach von «jener Frau» («that woman»), statt die Betroffene, Monica Lewinsky, beim Namen zu nennen, hätte dem aufmerksamen Zuhörer eine Warnung sein können. Im weiteren Verlauf der Lewinsky-Affäre stellte sich heraus, dass die Aussage Bill Clintons nicht der Wahrheit entsprach, zumindest dann nicht, wenn man Fellatio unter den Begriff der «sexual relations» fasst. Im Amtsenthebungsverfahren (impeachment) sprach ihn der Senat frei von den Vorwürfen des Meineids (perjury) und der Behinderung der Justiz (obstruction of justice). Jedoch wurde er in der Folge von einer Bundesrichterin für die Falschaussage im Verfahren Jones wegen Missachtung des Gerichts (civil contempt of court) verurteilt und mit USD 90'0oo Busse bestraft.

Zitiert nach Marc Uwe Kling, Das KänguruManifest, Berlin 2011, 136, zur Verlässlichkeit des Zitats, $67 \mathrm{ff}$.
3 Der Fall ist in mehrerlei Hinsicht instruktiv. Im Ergebnis zeigt er, dass selbst präsidiale Lügen kurze Beine haben und sie deshalb prozessual keine kluge Strategie sind. ${ }^{2}$ Die nachfolgenden Ausführungen sollen deshalb kein Plädoyer für die Lüge sein. Weiter zeigt die damals intensiv geführte Debatte um die Bedeutung des Begriffs «sexual relations», dass zwischen reiner Wahrheit und klarer Lüge oft ein beträchtlicher semantischer Interpretationsspielraum klafft. Auch wenn man sich darüber streiten kann, ob Bill Clinton zum Zeitpunkt seiner Fernsehansprache bereits ein Beschuldigter war und ob das Impeachment ein Strafverfahren im engeren Sinne ist, zeigt dieses Beispiel jedenfalls auf, welch drastischen Konsequenzen Lügen haben können. Lügen- und Ungehorsamsstrafen sind keineswegs nur ein Phänomen ferner Vergangenheit.

4 Auch die die Philosophie seit Langem beschäftigende Frage, was eine Lüge und wie sie zu bewerten ist, wird nicht weiter vertieft.3 Für die vorliegende Untersuchung wird die Lüge definiert als eine in Täuschungsabsicht und im Bewusstsein der Unwahrheit abgegebene Äusserung,

$2 \overline{\text { Das galt schon vor über hundert Jahren in einem }}$ sehr ähnlich gelagerten Fall, vgl. Arthur Kallmann, der leugnende Angeklagte, GA 1907, 230 ff., 232: «ein Mann war wegen eines Sittlichkeitsvergehens angeklagt, das, ein typisches Altersdelikt, der pathologischen Färbung wohl nicht entbehrte. Er leugnete vor dem Gericht wie vor seiner leidenden Frau; er mochte seine soziale Stellung retten, wirtschaftliche Nachteile abwenden, seiner Frau schädigenden Kummer fernhalten wollen, mochte hoffen, durch eigene Energie und die Einwirkung seiner Frau eine Wiederholung der Tat vermeiden zu können».

3 Jens Tobias Gruber, die Lüge des Beschuldigten im Strafverfahren, eine Untersuchung des deutschen Rechts unter Berücksichtigung des USamerikanischen Rechts des Bundes, Diss. Giessen 2007, Baden-Baden 2008, $37 \mathrm{ff}$. 
welche der Faktenlage objektiv widerspricht. Damit steht aber auch fest, dass gutgläubig für wahr gehaltene, in Wahrheit aber falsche Äusserungen ebenso wenig unter den Lügenbegriff fallen, wie Äusserungen, von denen der Sprechende weiss, dass sie objektiv falsch sind, er sie aber nicht in Täuschungsabsicht, sondern etwa ironisch äussert. 4

Lügen sind somit weder strategisch sinnvoll, noch in ihren Konsequenzen zu kontrollieren. Gleichwohl sind sie im Strafverfahren allgegenwärtig. Schweigen als Alternative zur Lüge scheint deshalb auf den ersten Blick die goldene Wahl zu sein. 5 Abgesehen davon, dass es sehr schwierig ist, auf - zumal strafrechtliche - Vorwürfe hin zu schweigen und nicht dem urmenschlichen Bedürfnis nachzugeben, sich mit (wahren oder unwahren) Aussagen zu erklären, ${ }^{6}$ spricht noch ein zweiter Grund gegen das Schweigen: Faktisch erzeugt «nichts so sehr den Anschein von Schuld wie die Aussage «no comment \!».7 Der Volksmund sagt:

\section{Gruber (Fn. 3), S. 25 ff.}

Dazu eingehend Stephan Bernard, Reden ist Silber, Schweigen ist Gold?, in: forumpoenale 2014, S. 2 ff.; diff. Peter Albrecht, Schweigen im Strafprozess, in: Hartmut von Sass (Hrsg.), Stille Tropen -: zur Rhetorik und Grammatik des Schweigens, Freiburg/München 2013, 156 f.

Albrecht (Fn. 5), S. 141.

7 Christoph Bentele, Zulässigkeit und Grenzen von Litigation-PR durch die Staatsanwaltschaft, Diss. Tübingen 2015, Marburg 2016, 46; in schonungsloser Offenheit auch der Einzelrichter am Bezirksgericht Zürich: «Indem die Angeklagte jegliche Aussage verweigert, benimmt sie sich zudem in einer für Angeklagte, denen Landfriedensbruch, Vermummung, Teilnahme an unbewilligten Demonstrationen etc. vorgeworfen wird, typischen Verhaltensweise». (NZZ Nr. 77 vom 2. April 2003, S. 43). Da hilft auch die nachgeschobene Erklärung nichts mehr, dass dies weder als konkretes Indiz noch irgendwie verschuldensrelevant zu werten sei, es ergäben sich aus dem Schweigen aber eben auch keinerlei entlastende Momente.
«Keine Antwort ist auch eine Antwort» 8 . «Beredtes Schweigen» ist kein kommunikatives Nichts, sondern eine Stellungnahme ohne Worte, welche auch Erwartungen enttäuscht, zumal Schweigen bei den Strafbehörden zu Mehrarbeit führt. 9 Während im Zivilrecht seit Langem anerkannt ist, dass eine Stellenbewerberin, welche auf die Frage ihres künftigen Arbeitgebers, ob sie schwanger sei, schweigt, den Anschein erweckt die Frage zu bejahen und ihr deshalb das Recht zusteht, eine bestehende Schwangerschaft wahrheitswidrig in Abrede zu stellen, ${ }^{10}$ tut man sich im Strafverfahren nach wie vor schwer, ein Recht zur Lüge zu akzeptieren. ${ }^{11}$ Und dies, obwohl in der Sache unbestritten ist, dass Schweigen de facto als Zustimmung zur Anklage gedeutet wird und insoweit auch im Strafverfahren «qui tacet consentire videtur» ${ }^{12}$ gilt.

8 Regula Schlauri, Das Verbot des Selbstbelastungszwangs im Strafverfahren - Konkretisierung eines Grundrechts durch Rechtsvergleichung, Diss. Zürich 2003, S. 317.

9 So überzeugend Albrecht (Fn. 5), S. 142 ff.

10 Emmel in Amstutz/Breitschmid/Furrer/Girsberger/Huguenin/ Jungo/Müller-Chen/Roberto/ Schnyder/Trüeb (Hrsg.), Handkommentar zum Schweizer Privatrecht, 3. Aufl., Zürich 2016, Art. $320 \mathrm{~N} \mathrm{1}$; Kirsten Schmalenbach, Wahrheit und Lüge unter der Herrschaft der Grundrechte, Juristische Arbeitsblätter 2005, S. 751 m.w.H.

11 Niklaus Schmid/Daniel Jositsch, Handbuch des schweizerischen Strafprozessrechts, 3. Aufl., Zürich/St. Gallen 2017, N 159 f. («Die Frage kann jedoch offen bleiben.»); vgl. auch Yvan Jeanneret/André Kuhn, Précis de procédure pénale, $2^{\mathrm{e}}$ édition, Berne 2018, N. 4064; Niklaus Oberholzer, Grundzüge des Strafprozessrechts, 3. Aufl., Bern 2012, N 418 ff.; Niklaus Schmid/Daniel Jositsch, Schweizerische Strafprozessordnung, Praxiskommentar, 3. Aufl., Zürich/St. Gallen 2018 (fortan zit.: PK-StPO), Art. $113 \mathrm{~N} 1$.

12 Schlauri (Fn. 8), 317; zu dieser zivilrechtlichen Rechtsparömie vgl. Max Baumann, in: Gauch/Schmid (Hrsg.), Kommentar zum Schweizerischen Zivilgesetzbuch, Einleitung, Das Personenrecht, Einleitung, Art. 1-7 ZGB, 3. Aufl. Zürich 1998, Art. 2 N 94 ZGB. 
6 Es lohnt sich deshalb, die Lüge im Strafverfahren - wieder einmal ${ }^{13}$ - etwas genauer in den Blick zu nehmen. Die hier interessierende Frage, ob der Beschuldigte lügen darf, wird in vier Schritten beantwortet. Zunächst wird gezeigt, dass er ein Recht hat zu schweigen (II.). Daran schliesst die Frage an, ob ihn, für den Fall, dass er aussagt, eine Wahrheitspflicht (III.) trifft, er die Vorwürfe abstreiten darf (IV.) oder er sogar ein Recht zur Lüge hat (V.).

\section{Schweigerecht}

7 Noch in der ersten Hälfte des 19. Jahrhunderts galt im zürcherischen Strafverfahren eine Aussagepflicht. Bei Aussageverweigerung drohte das sprichwörtliche Wasser und Brot. § 86 des Gesetzes über die Strafrechtspflege des Kantons Zürich vom 10. Juni $1831^{14}$ bestimmte:

13 Vgl. bereits: Karl Binding, Die Wahrheitspflicht im Prozess, DJZ 1909, S. 162 ff.; Wilfried Bottke, Wahrheitspflicht des Strafverteidigers, ZStW 96/1984, Heft 3, S. 726 ff.; Albin Eser, Aussagefreiheit und Beistand des Verteidigers im Ermittlungsverfahren Rechtsvergleichende Beobachtungen zur Rechtsstellung des Beschuldigten, Sonderdruck des Originalbeitrags erschienen in: Zeitschrift für die gesamte Strafrechtswissenschaft: nebst Auslandsteil 17 (1967), S. 213=565 - 271=623; ders., Der Schutz vor Selbstbezichtigung im deutschen Strafprozessrecht, Originalbeitrag erschienen in: Deutsche strafrechtliche Landesreferate zum ... Internationalen Kongress für Rechtsvergleichung, 9 (1974), S. [136] - 171; Gerhard Fezer, Hat der Beschuldigte ein Recht auf Lüge?, in: Küper/Welp (Hrsg.), Beiträge zur Rechtswissenschaft, Festschrift für Walter Stree und Johannes Wessels zum 70. Geburtstag, Heidelberg 1993, 663 ff.; Gruber (Fn. 3), passim; Kallmann (Fn. 2), S. 230 ff.; Heribert Ostendorf, Strafvereitelung durch Strafverteidigung, Zur Diskussion um Gründe und Leitbild berufsmäßiger Strafverteidigung, NJW 1978, S. 1345 ff.; Edmund Mezger, Die Beschuldigtenvernehmung auf psychologischer Grundlage, ZStW 40/1919, S. 152 ff.; Hans Felix Pfenninger, Die Bekämpfung der Lüge im Strafverfahren, SJZ 1961, S. 53 ff.; ders. Die Wahrheitspflicht des Beschul-
«Wenn aber ein von dem Criminalgericht bereits in Anklagezustand versetzter Gefangener sich weigert, bestimmte Antworten zu geben, ... so kann derselbe mit Verschärfung der Haft, mit Fesseln und Schmälerung der Kost bestraft werden». Ganz wohl scheint es dem Gesetzgeber damit aber nicht gewesen zu sein, wie ein Blick auf $\S 87$ zeigt: «Dieß soll indessen nur auf den Antrag des Cantonalverhöramts, nach eingehohltem Gutachten des Staatsanwalds und in Folge Beschlusses des Criminalgerichts geschehen dürfen: dieser Beschluß soll genau bestimmen, welche der angegebenen Strafen und in welchem Grade sie Statt finden soll».

8 Heute ist vollkommen unbestritten, dass der Beschuldigte ein Recht hat, zu schweigen. ${ }^{15}$ Die beschuldigte Person

digten im schweizerischen Strafverfahren, SJZ 1957, S. 129 ff. und 145 ff.; Hinrich Rüping, Zur Mitwirkungspflicht des Beschuldigten und Angeklagten, Juristische Rundschau (JR), Berlin 1974, S. 135 ff.; Franz Salditt, Das Interesse an der Lüge, Strafverteidiger (StV) 1999, S. 61; Schmalenbach (Fn. 10), S. 749 ff.; Richard Schmidt, Die Lüge im Prozess, DJZ 1909, S. 39 ff.; Eberhard Schorsch, Phantasie, Irrtum, Lüge und die Wahrheitsfindung, StV 1985, S. 522 ff.; Hans Vest, Das Beweisantragsrecht des Beschuldigten, in: Donatsch/Forster/Schwarzenegger (Hrsg.), Strafrecht, Strafprozessrecht und Menschenrechte, Festschrift für Stefan Trechsel zum 65. Geburtstag, Zürich/Basel/Genf 2002, S. 781 ff., S. 793; Hans Walder, Die Vernehmung des Beschuldigten, dargestellt am Beispiel des zürcherischen und deutschen Strafprozessrechts, Habil. Zürich 1965, Hamburg 1965; Johannes Wessels, Schweigen und Leugnen im Strafverfahren, Juristische Schulung (JuS) 1966, S. 169 ff.; Wolfgang Wohlers, Strafverteidigung vor den Schranken der Strafgerichtsbarkeit, StV 2001, S. $420 \mathrm{ff}$.

14 Gesetz über die Strafrechtspflege des Kantons Zürich vom 10. Juni 1831, StA ZH OS 1 (S. 177211).

15 Albrecht (Fn. 5), S. 145 f.; Jeanneret/Kuhn (Fn. 11), N. 4064; Oberholzer (Fn. 11), N 418; Mark Pieth, Schweizerisches Strafprozessrecht, 3. Aufl., Basel 2016, 88 f.; Schmid/Jositsch (Fn. 11), N 671 ff. zu den Grundlagen unter altem Recht Flachsmann Stefan/Wehrenberg Stefan, Aussa- 
muss sich nicht selbst belasten. Sie hat namentlich das Recht, die Aussage und ihre Mitwirkung im Strafverfahren zu verweigern (Art. 113 Abs. 1 StPO). Im ersten Satz dieser Bestimmung wird der Grundsatz «nemo tenetur se ipsum accusare» gesetzlich verankert, der auch als «privilege against self-incrimination» 16 oder als Selbstbelastungsfreiheit 17 bezeichnet wird. Dieses Prinzip wird auch aus übergeordnetem Recht hergeleitet. So etwa aus Art. 14 Ziff. 3 lit. g UNO-Pakt II, wonach ein Angeklagter nicht gezwungen werden darf, gegen sich selbst als Zeuge auszusagen. ${ }^{18}$ Es ergibt sich ferner aus dem Recht auf ein faires Verfahren (Art. 6 Abs. 1 EMRK) ${ }^{19}$ und aus der Unschuldsvermutung (Art. 6 Abs. 2 EMRK). ${ }^{20}$ Der zweite Satz von Art. 113 Abs. 1 StPO gewährt dem Beschuldigten unter anderem ein Aussageverweigerungsrecht.

9 Den Beschuldigten trifft somit keine Aussagepflicht. Eine Aussagepflicht ergibt sich auch nicht aus Art. 143 Abs. 4 StPO, wonach die Strafbehörde die einzuvernehmende Person auffordert, sich zum

geverweigerungsrecht und Informationspflicht, SJZ 97 (2001) 313, S. 314 ff.

16 Eser, Schutz vor Selbstbezichtigung (Fn. 13), S. 140.

17 Viktor Lieber, in Donatsch/Hansjakob/Lieber (Hrsg.), Kommentar zur Schweizerischen Strafprozessordnung (StPO), 2. Aufl., Zürich 2014 (fortan zit.: ZHK StPO²-Bearbeiter), Art. $113 \mathrm{~N} 1$.

18 BGE 142 IV 207, E. 8.2.

19 BGE 142 IV 207, E. 8.3; für die h.L. vgl. nur Dominique Ott, Der Grundsatz «nemo tenetur se ipsum accusare», unter besonderer Berücksichtigung der strassenverkehrsrechtlichen Pflichten, Diss. Zürich 2012, Zürich/Basel/Genf 2012, S. 106 f.; a.M. Hansjörg Seiler, Das (Miss-) Verhältnis zwischen strafprozessualem Schweigerecht und verwaltungsrechtlicher Mitwirkungsund Auskunftspflicht, recht 1/2005, S. 11 ff., 20.

20 Urteil des EGMR 19187/91 vom 17.12.1996 (Saunders $v$. The United Kingdom), Ziff. 68; offen gelassen in BGE 131 I 272, Erw. 3.2.3.2;
Gegenstand der Einvernahme zu äussern. Diese Bestimmung dient nur dazu «der einvernehmenden Person Richtlinien für die Gesprächsführung an die Hand» zu geben. ${ }^{21}$ Teilweise wird vertreten, dass der Beschuldigte zwar nicht verpflichtet ist, zur Sache auszusagen, ihn jedoch eine Pflicht treffe, zu seiner Person Auskunft zu geben. ${ }^{22}$ Unbestrittenermassen ist eine solche Aussagepflicht dann abzulehnen, wenn sich der Beschuldigte bereits durch die Angabe der Personalien selber belastet (z.B. Ausländereigenschaft bei Delikten nach dem Ausländergesetz). 23 Eine Pflicht zur Auskunft über die Personalien ist aber auch im Übrigen kategorisch abzulehnen. Nicht nur fehlt eine entsprechende Gesetzesgrundlage,24 die Auskunftspflicht widerspricht auch übergeordnetem Recht. Der Nemo-tenetur-Grundsatz entbindet den Beschuldigten ganz grundsätzlich davon, aktiv an seiner Überführung mitzuwirken. Die Behörden haben genügend Möglichkeiten, die Identität oder das Einkommen des Beschuldigten selber zu eruieren.

10 Der Beschuldigte hat also ein Recht zu schweigen. Daraus folgt indessen noch nicht, dass in seinem Aussageverhalten völlig frei ist, wer auf sein Schweigerecht verzichtet. Es stellt sich mit anderen Worten die Frage, ob der Beschuldigte, wenn er aussagt, eine Pflicht hat, die Wahrheit zu sagen.

21 ZHK StPO2-Godenzi, Art. 143 N 29; vgl. auch PKStPO (Fn. 11), Art. 143 N 13.

22 Nachweise bei Albrecht (Fn. 5), S. 145 und ZHK StPO'-Lieber, Art. $113 \mathrm{~N} 17$.

23 Überzeugend ZHK StPO²-Godenzi, Art. 143 $\mathrm{N} 21 \mathrm{ff}$.

24 Skeptisch bereits Felix Bommer, Parteirechte der beschuldigten Person bei Beweiserhebungen in der Untersuchung, recht 2010, S. 196 ff., 199 dortige Fn. 17. 


\section{Wahrheitspflicht}

11 Der neapolitanische Strafrechtsphilosoph Gaetano Filangieri hat schon 1782 darauf hingewiesen, dass der Grund, weshalb im aufgeklärten Strafprozess auf eine Wahrheitspflicht verzichtet werden sollte, darin liegt, dass man den Beschuldigten vor dem Dilemma bewahren möchte, sich entweder durch eine wahrheitsgemässe Aussage selber aufs Schafott zu bringen oder bei einer Falschaussage wegen Meineids bestraft zu werden. ${ }^{25}$ Gleichwohl ging die Prozesslehre noch lange von «lex imperfecta» aus, wonach eine Wahrheitspflicht ohne Wahrheitszwang bestehe. ${ }^{26}$

Hans Felix Pfenninger hat aufgezeigt, dass dem Inquisitionsprozess die Idee zugrunde lag, «es sei der Beschuldigte zur wahrheitsgemäßen Aussage nach göttlichem und menschlichem Recht verpflichtet» ${ }^{27}$. Wegen dieser tiefen Verwurzelung der Wahrheitspflicht und weil es für eine Überführung des Inquisiten entweder seines Geständnisses oder der Aussage zweier Zeugen bedurfte, wurde noch weit über die Abschaffung der Folter hinaus am Geständniszwang festgehalten. Das Geständnis wurde nunmehr lediglich mittelbar über Lügen- und Ungehorsamsstrafen abgesichert. ${ }^{28}$ Erst allmählich setzte sich die Einsicht durch, dass die freie richterliche Beweiswürdigung als prozessuales Gegenstück an die Stelle der abgeschafften Folter treten sollte und damit zugleich Geständnis- zwang und Wahrheitspflicht überflüssig wurden. ${ }^{29}$

13 Der auf diesen Einsichten aufbauende reformierte Strafprozess kommt deshalb folgerichtig ohne eine Wahrheitspflicht des Beschuldigten aus. Die Wahrheitssuche erfolgt in diesem Prozess mittels Zeugen und Sachbeweisen. Die Einvernahme des Beschuldigten selbst hat zwar im strafprozessualen Alltag ein enormes Gewicht, 30 ist als Mittel der Wahrheitsfindung indes «strukturell ohne Bedeutung»31. Nur schon aus dem Schweigerecht ergibt sich, dass die Strafprozessordnung phänomenologisch darauf angelegt ist, die Wahrheit ohne Beteiligung des Beschuldigten zu eruieren. $3^{2}$ Eindringlich bringt dies der 5. Zusatz zur Verfassung der Vereinigten Staaten von Amerika zum Ausdruck: «No person ... shall be compelled in any criminal case to be a witness against himself». Über Art. 14 Ziff. 3 lit. g UNO-Pakt II («Not to be compelled to testify against himself») ist dieser Grundsatz auch für die Schweiz verbindlich.

14 Damit wird nicht verkannt, dass die Einlassung des Beschuldigten und insbesondere sein Geständnis auch im heutigen Strafverfahren eine absolut überragende Bedeutung haben.33 Dies gilt in besonderem Mass für die zahlenmässig absolut dominierenden Erledigungsformen, welche de facto kaum (Strafbefehlsverfahren) oder de iure nicht (abgekürztes Ver-
$25 \overline{\text { Gaetano Filangieri, la scienza della legislazione, }}$ Vol. II, Firenze 1782, S. 87.

26 M.w.H. Wessels (Fn. 13), S. 173.

27 Pfenninger, SJZ 1957 (Fn. 13), S. 130.

28 Wessels (Fn. 13), S. 170; Pfenninger, SJZ 1957 (Fn. 13), S. 131; Kallmann (Fn. 2), S. 230.
Pfenninger, SJZ 1957 (Fn. 13), S. 133.

30 Zur Bedeutung der Mitwirkung im Allg. Albrecht (Fn. 5), S. 142.

$31 \quad$ Fezer (Fn. 13), S. 669.

32 Gl. Pfenninger, SJZ 1957 (Fn. 13), S. 146 ff.

33 Das sieht auch Fezer (Fn. 13), S. 669. 
fahren) ohne Geständnis auskommen. ${ }^{34}$ Wohl nicht zufällig hat der Gesetzgeber der schweizerischen Strafprozessordnung im 4. Titel zu den Beweismitteln (Art. 139 ff. StPO) die Einvernahme der beschuldigten Person (Art. 157 ff. StPO) an den Kapitelanfang gesetzt. Indem die Beschuldigteneinvernahme jedoch systematisch als Beweismittel eingeordnet wird, fällt die schweizerische Strafprozessordnung in einem zentralen Punkt der prozessualen Wahrheitsfindung hinter den Erkenntnisstand des reformierten Strafprozesses zurück. Selbst wenn man aber mit der (hierzulande wohl herrschenden35) Lehre davon ausgeht, dass die Aussage des Beschuldigten als Beweismittel gilt, ändert dies nichts daran, dass der Beschuldigte weder mit Gewalt zur Wahrheit gezwungen werden kann (Art. 140 StPO), ${ }^{6}$ noch irgendeine Bestimmung ihn verpflichtet, bei einer freiwilligen Aussage die Wahrheit zu offenbaren. In der Lehre fordert soweit ersichtlich niemand mehr ernsthaft eine Wahrheitspflicht des Beschuldigten. ${ }^{37}$

Im Grundsatz schliesst sich dem auch das Bundesgericht an. Im Detail ist die bundesgerichtliche Rechtsprechung in Bezug auf die Wahrheitspflicht jedoch nicht eindeutig. Ein Einzelrichter hatte einen Beschuldigten explizit darauf hin-

34 Marc Thommen, kurzer Prozess - fairer Prozess? Strafbefehls- und abgekürzte Verfahren zwischen Effizienz und Gerechtigkeit, Habil. Luzern, Bern 2013, S. $64 \mathrm{ff}$. und $163 \mathrm{ff}$.

35 Vgl. nur Niklaus Ruckstuhl, in Niggli/Heer/ Wiprächtiger (Hrsg.), Schweizerische Strafprozessordnung/Jugendstrafprozessordung

(StPO/JStPO), 2. Aufl., Basel 2014 (fortan zit.: BSK StPO2-Bearbeiter), Art. 157 N 1.

36 BSK StPO2-Gless, Art. $140 \mathrm{~N} 32$.

37 Cornel Borbély, Die Kostentragung in Einstellungsverfügungen, ZStrR 129/2011, S. 415 ff., 430; BSK StPO ${ }^{2}$-Ruckstuhl, Art. $157 \mathrm{~N}$ 1a, anders nur noch Walder, Habil. (Fn. 13), S. 72. gewiesen, dass er nicht zur Aussage verpflichtet sei, ihn aber ermahnt, wenn er etwas sage, solle es die Wahrheit sein. Das sei auch Ehrensache. Hierzu hält das Bundesgericht zunächst generell fest, dass der Beschuldigte im Strafverfahren keiner Wahrheitspflicht unterliege. Dann stuft es die einzelrichterliche Ermahnung jedoch als bundesrechtskonform und die Aussage des Beschuldigten deshalb als verwertbar ein. Aus der Tonaufnahme der Hauptverhandlung ergebe sich, dass es sich «um eine moralische Ermahnung und keine gesetzliche Verpflichtung handelte». Aufgrund der gesamten Belehrung habe beim Beschuldigten nicht der Eindruck entstehen können, «er sei für den Fall, dass er aussage, zur Wahrheit verpflichtet».38 Abgesehen davon, dass das Bundesgericht schon von Verfassungs wegen (Art. $188 \mathrm{ff}$. BV) nur für Fragen des Rechts und nicht der Moral letzte Instanz ist, 39 überzeugt seine Begründung auch inhaltlich nicht. Aus der Ermahnung, dass eine wahrheitsgemässe Aussage «auch» Ehrensache sei, konnte der Beschuldigte sehr wohl ableiten, dass unwahre Aussagen zusätzlich rechtliche Folgen haben.

16 In einem anderen Entscheid wurden die Mitbeschuldigten in einer Konfrontationseinvernahme zunächst auf ihr Recht hingewiesen, Aussagen und Mitwirkung $\mathrm{zu}$ verweigern. Im Anschluss daran forderte sie der verfahrensleitende Staatsanwalt auf, die Wahrheit zu sagen, und machte sie auf die Strafbarkeit der falschen Anschuldigung, der Irreführung der Rechtspflege und der Begünstigung gemäss Art. 303-305 StGB aufmerk-

38 Urteil des Bundesgerichts 6B_604/2012, 6B_613/2012 vom 16. Januar 2014, E. 3.4.4.

39 Bereits Kallmann (Fn. 2), S. 232. 
sam..$^{\circ 0}$ Auch darin sah das Bundesgericht keine Bundesrechtsverletzung. Der Beschuldigte habe das Recht, die Aussage und seine Mitwirkung im Strafverfahren zu verweigern (Art. 113 Abs. 2 StPO) und er unterliege keiner Wahrheitspflicht. Der Beschwerdeführer sei nicht auf eine Pflicht zur wahrheitsgemässen Aussage hingewiesen, sondern lediglich ermahnt worden, für den Fall, dass er aussage, die Wahrheit zu sagen. Die Aufforderung oder Ermahnung, die Wahrheit zu sagen, sei nicht gleichbedeutend mit einem Hinweis auf die Wahrheitspflicht. Es handle sich hierbei lediglich um eine moralische Ermahnung, welche die Bedeutung und den Ernst der Befragung unterstreichen soll. Ferner sei die Ermahnung zur Wahrheit im Kontext mit dem Hinweis auf die Rechtspflegedelikte zu sehen. ${ }^{41}$

Auch in diesem Urteil überzeugt lediglich, dass zunächst eine Wahrheitspflicht kategorisch verneint wird. Nicht überzeugend ist indes, dass das Vorgehen der Strafbehörden bundesrechtskonform gewesen sein soll. Entscheidend ist, ob beim Beschuldigten aufgrund der gesamten Belehrung der Eindruck entsteht, er sei zur Wahrheit verpflichtet.42 Entgegen dem Bundesgericht wurde der Mitbeschuldigte vorliegend keineswegs nur an seine (wiederum nicht näher begründete) moralische Pflicht zur Wahrheit erinnert, sondern vielmehr aufgefordert, die Wahrheit zu sagen, gefolgt von einem Hinweis auf drohende Straffolgen bei unwahrer Aussage. Auf den Punkt gebracht, ist ihm in der Belastungssituation

40 Urteil des Bundesgerichts 6B_157/2016 vom 8. August 2016, E. 2.4.1.

41 Urteil des Bundesgerichts 6B_157/2016 vom 8. August 2016, E. 2.4.2.

42 Überzeugend ZHK StPO²-Lieber, Art. $113 \mathrm{~N}$ 13b. der Einvernahme gesagt worden, er solle die Wahrheit sagen, sonst drohe ihm eine Strafe. Inwiefern eine solche Aufklärung beim Beschuldigten nicht den Eindruck geweckt haben soll, er sei sogar unter Strafandrohung zur Wahrheit verpflichtet, ist nicht nachvollziehbar. Dass er «nicht auf eine Pflicht zur wahrheitsgemässen Aussage hingewiesen» worden sei, ist somit nicht nur wortklauberisch, sondern schlicht falsch.

18 Heute wird eine Wahrheitspflicht zu Recht verneint. Eine Ermahnung zur Wahrheit ist deshalb nicht haltbar, 43 auch keine moralische. Der Beschuldigte hat somit nicht nur keine Pflicht, auszusagen, ihn trifft auch für den Fall, dass er aussagt, keine Wahrheitspflicht. Daran schliesst unweigerlich die Frage an, ob er die Vorwürfe leugnen (sogleich IV.) oder sogar lügen (V.) darf.

\section{Recht zur Leugnung}

19 Dem Beschuldigten steht unbestrittenermassen das Recht zu, die Aussage zu verweigern. Dennoch ist Schweigen meist eine wenig attraktive Option, weil es intuitiv als Schuldeingeständnis gewertet wird. Seit Langem wird darauf hingewiesen, dass das Schweigerecht ein «privilegium odiosum» darstelle, da es selbst für professionelle Strafbehörden de facto eine «psychologisch unmögliche Forderung» darstelle, Schweigen nicht zulasten des Angeklagten zu werten. 44 Wenn Nichtssagen keine Option ist, darf der Beschuldigte dann durch Reden nichts sagen?

43 Z.R. Gruber (Fn. 3), S. 298.

44 Beide Zitate aus Pfenninger, SJZ 1957 (Fn. 13), S. $146 \mathrm{f}$. 
20 Bevor wir uns unten dem Recht zur Lüge widmen, soll hier darauf eingegangen werden, ob Leugnen erlaubt ist. Terminologisch wird unterschieden zwischen «blossem» Leugnen respektive «einfachem» Bestreiten einerseits und «qualifiziertem» Lügen andererseits. 45 Dass sich Leugnen und Lügen nicht trennscharf abgrenzen lassen, zeigt bereits das Eingangsbeispiel von Bill Clinton, der die gegen ihn erhobenen Vorwürfe bestritt, indem er explizit versicherte, er habe mit Monica Lewinsky keine sexuellen Beziehungen unterhalten. Darf der Beschuldigte den Tatvorwurf wahrheitswidrig abstreiten, also sagen, er sei nicht der Täter, oder behaupten er sei unschuldig?

In Deutschland wurde ein Recht zur Leugnung seit Langem ${ }^{46}$ und letztlich mit Erfolg postuliert. So hielt das Bayerische Oberste Landgericht 1985 fest, sei es das «gute Recht des Angeklagten, die Tatbegehung zu leugnen»47. Der Bundesgerichtshof entschied wenige Jahre später, die explizite Behauptung, eine Tat nicht begangen zu haben, sei dem Schweigen

45 BGE 103 IV 8, Regeste («einfaches Leugnen»), E. 3b («einfache Bestreitungen»); Schmid/ Jositsch (Fn. 11), Handbuch des schweizerischen Strafprozessrechts, 2. Aufl., Zürich/St. Gallen 2013, N 1789 Fn. 81 («qualifizierte Lügen»); schon Kallmann (Fn. 2), S. 232 f. («kühn Erdichtetes») und Wessels (Fn. 13), 173; kritisch zur Unterscheidung, Fezer (Fn. 13), S. 679 und Wolfgang Frisch, Gegenwärtiger Stand und Zukunftsperspektiven der Strafzumessungsdogmatik, Das Recht der Strafzumessung im Lichte der systematischen Darstellungen von HansJürgen Bruns und Franz Pallin (Teil II), ZStW 99/1987, S. 751 ff., 782; zur vorliegend verwendeten Definition der Lüge als eine in Täuschungsabsicht und im Bewusstsein der Unwahrheit abgegebene Äusserung, welche der Faktenlage objektiv widerspricht, vgl. oben bei Fn. 4.

46 Kallmann (Fn. 2), S. 231.

47 Urteil des Bayrischen Oberlandgerichts (1. Strafsenat), Beschluß vom 21. 5. 1985 - RReg. 1 St 73/85, E. 1a; s.a. Fezer (Fn. 13), S. 675; Kallmann (Fn. 2), S. 231. gleichzusetzen. $4^{8}$ Leugnen wurde deshalb auch schon als qualifiziertes Schweigen bezeichnet.49 Dagegen wurde überzeugend eingewendet, dass Leugnen und Schweigen nicht das Gleiche ist. $5^{\circ}$ Es ist deshalb zweifelhaft, ob man ein Recht zur Leugnung aus dem Nemo-teneturGrundsatz ableiten kann. .1 $^{1}$

22 Das schweizerische Bundesgericht hat in einem Entscheid betreffend die Anrechnung von Untersuchungshaft - differenzierend festgehalten, dass die Leugnung von Vorwürfen zwar über die Verweigerung von Auskünften hinausgehe, im Ergebnis Bestreiten und Schweigen jedoch gleich zu bewerten seien. Einfache Bestreitungen hätten auf den Gang und die Dauer des Verfahrens keine weitergehenden Auswirkungen als die Aussageverweigerung. Beschränke sich der Beschuldigte darauf, die ihm vorgeworfenen Straftaten zu bestreiten, versage er eine Mitwirkung, zu der er nicht verpflichtet sei. «Das Verfahren wird dadurch noch nicht erschwert oder verlängert, denn die Lügen des Beschuldigten schmälern nicht die Beweiskraft der gesammelten Beweise».52 In einem neueren Entscheid betreffend eine Falschanschuldigung hat das Bundesgericht festgehalten, dass das Leugnen der eigenen Täterschaft und das «blosse» Bestreiten belastender Aussagen straflos seien, auch wenn dadurch der Verdacht notwendig auf eine andere Person gelenkt werde, nicht hingegen das Bezichti-

$4 8 \longdiv { \text { Beschluss des Bundesgerichtshofs } 1 \text { StR 196/92 } }$ vom 17 Juni 1992; dagegen wendet Gruber ([Fn. 3), S. $267 \mathrm{ff}$. ein, dass «Leugnen kein Schweigen» ist, im Ergebnis jedoch gl. M.

49 Eser, Aussagefreiheit (Fn. 13), S. 213 ff., 221 dortige Fn. 35.

50 Gruber (Fn. 3) 114 und 264 ff.

51 Dazu sogleich unten Ziff. 5 .

52 BGE 103 IV 8, E. 3b. 
gen einer anderen Person.53 Zusammengefasst behandelt die Rechtsprechung Leugnen und Schweigen gleich. Der Beschuldigte darf den Tatvorwurf somit leugnen und seine Täterschaft abstreiten.

\section{Recht zur Lüge}

23 Wenn man eine Wahrheitspflicht des Beschuldigten verneint, dann stellt sich unweigerlich die Frage, ob im Strafverfahren ein Recht zur Lüge54 besteht.55 Die ältere Lehre hat ein Recht zur Lüge kategorisch verneint. Es gehöre Kälte dazu, einem Menschen ein Recht auf Lüge überzustülpen. ${ }^{6}$ Berühmt geworden ist der Ausspruch des Grafen zu Dohna, ihm sei «unerfindlich, wie jemand aus der Begehung eines Verbrechens sollte das Recht herleiten dürfen, das Gericht $\mathrm{zu}$ belügen»57. Es wurde sogar vertreten, dass die Verpflichtung zur Sühne nicht erst mit seiner Strafe beginne, sondern bereits im Strafverfahren ein wahrheitsgemässes Schuldbekenntnis erfordere. 58 Die letzten beiden Standpunkte kranken freilich schon an einer petitio principii, indem sie die im Strafverfahren erst zu beweisende Schuld als bereits erwiesen unterstellen. Um ein Lügerecht zu verneinen, wird nicht selten auch Immanuel Kant bemüht, nach dem die Lüge die Vernichtung der Menschenwürde des

53 BGE 132 IV 20.

54 Teilweise ist auch von einem «Recht auf Lüge» die Rede, so insb. Fezer (Fn. 13), S. 663 ff. oder ZHK StPO2-Lieber, Art. $113 \mathrm{~N}$ 29. Weil es aber nicht um den Anspruch geht, angelogen zu werden, sondern um die Berechtigung im Strafverfahren Unwahres zu äussern, sollte von einem Recht zur Lüge gesprochen werden.

55 A.A. Beschluss des Bundesgerichtshofs 5 StR 328/04 vom 17. 3. 2005 (LG Hildesheim), NStZ 2005, S. 517 ff., 518.

56 Salditt (Fn. 13), S. 63.

57 Hier zitiert nach Wessels (Fn. 13), S. 173.

58 Henkel hier zitiert nach Wessels (Fn. 13), S. 173.
Lügners in seiner eigenen Person sei.59 Dabei wird zuweilen allerdings unterschlagen, ${ }^{60}$ dass die Lüge nach Kant nicht einer Rechts-, sondern «bloss» einer Tugendpflicht auf eigene Vervollkommnung61 zuwiderläuft. ${ }^{62}$ Teilweise wird auch utilitaristisch argumentiert, dass ein Lügerecht letztlich die Position des Beschuldigten im Prozess unterwandere,63 oder aus Sicht der Strafverfolgung hervorgehoben, dass Lügen die Wahrheitsfindung aktiv obstruieren.64 Klar dominierend war jedoch lange Zeit die moralische Argumentation.65 Es war die Rede vom «hartnäckigen» Schweigen und «frechen» Lügen des Beschuldigten. ${ }^{66}$ Es wurde sogar vertreten, der Beschuldigte sei nicht berechtigt zu lügen, weil er damit die «dem Gericht von Rechts wegen, nicht bloss als moralische Pflicht geschuldete Achtung» verletze. ${ }^{67}$

24 Heute verneint die deutsche Rechtsprechung ein Recht zur Lüge nach wie vor kategorisch. Dass keine Wahrheitspflicht bestehe, bedeute nicht, dass es ein Recht zur Lüge gebe. ${ }^{68}$ Gemäss dem schweizerischen Bundesgericht könne «ein allfälliges Recht zu lügen nur innerhalb der

$59 \overline{\text { Immanuel Kant, Metaphysik der Sitten, Zweiter }}$ Teil. Metaphysische Anfangsgründe der Tugendlehre, Kapitel zur Pflicht des Menschen gegen sich selbst, bloß als einem moralischen Wesen, 1. Von der Lüge, § 9.

60 So etwa Salditt (Fn. 13), S. 63.

61 Zur Unterscheidung von Rechts- und Tugendpflichten Kurt Seelmann, Rechtsphilosophie, München 1994, § 3 N 15.

62 Z.R. diff. Gruber (Fn. 3), S. 51 ff. und Schmalenbach (Fn. 10), S. 749

63 Salditt (Fn. 13), S. 63; Kallmann (Fn. 2), S. 233.

64 Eser, Aussagefreiheit (Fn. 13), S. 221/573, Fn. 35; Kallmann (Fn. 2), S. 233.

65 Wessels (Fn. 13), S. 173; Eser, Schutz vor Selbstbezichtigung (Fn. 13), S. 136 ff., $140 \mathrm{f}$.

66 Walder, Habil. (Fn. 13), S. 215.

67 Mezger (Fn. 13), S. 152 ff. 162.

68 Urteil des Bundesgerichtshofs 1 StR 488/14 vom 10. Februar 2015, NJW 2015, S. 1705 f., 1706. 
Grenzen des Strafrechts Geltung erlangen».69 Ob ein Recht zur Lüge besteht, wird damit offengelassen. Auch die Lehre zeigt sich grösstenteils zögerlich.70 Es möge «rechtsethisch gesehen kein Recht zur Lüge geben», doch bleibe die Lüge ohne direkte strafprozessuale Sanktion. Vorbehalten blieben indes falsche Anschuldigungen oder die Irreführung der Rechtspflege. ${ }^{71}$ Es sei umstritten, ob ein Recht auf Lüge bestehe, weil aber an die einfache Lüge keine unmittelbaren Sanktionen geknüpft werden dürften, bleibe die Frage ohne praktische Relevanz. ${ }^{72}$ Es bestehe zwar keine Wahrheitspflicht, aber auch kein Recht zur Lüge.73 Dabei bleibt ebenso offen, was es bedeuten soll, dass der Beschuldigte «rechtsethisch» kein Recht zur Lüge habe, wie ob es zutrifft, dass an die Lüge keine Sanktionen geknüpft werden.

Der Beschuldigte hat ein Recht zur Lüge. Wenn man eine Wahrheitspflicht verneint und sogar die Leugnung von Tatvorwürfen für das «gute Recht» des Beschuldigten hält, dann ist nicht nachvollziehbar, weshalb man ihm nicht auch ein Recht zur Lüge zugestehen will. Solche Hemmungen können wohl nur damit er-

$69 \overline{\text { Urteil des Bundesgerichts 6B_157/2016 vom }}$ 8. August 2016, E. 2.4.2.

70 Schmid/Jositsch (Fn. 11), N 159 f.; PK-StPO (Fn. 11), Art. $113 \mathrm{~N}$ 1; s. auch Jeanneret/Kuhn (Fn. 11), N 4064; Oberholzer (Fn. 11), N 418 ff.; eindeutig immerhin Borbély (Fn. 37), S. 430.

${ }_{71}$ BSK StPO2-Engler, Art. $113 \mathrm{~N}$ 6; insb. in Bezug auf die Irreführung der Rechtspflege ist dies freilich umstritten, zumal eine Falschaussage, welche «lediglich die staatliche Wahrheitsfindung behindert» nach überzeugender Ansicht straflos sein muss, vgl. Schmalenbach (Fn. 10), S. 751.

72 ZHK StPO-2-Lieber, Art. 113 N 29; Niklaus Schmid, Strafprozessrecht, 4. Aufl., Zürich 2004, $\mathrm{N} 272$.

73 Monka, in Graf (Hrsg.), BeckOK stopp mit RistBV und MiStra, 29. Aufl., Stand 1. Januar 2018, § 136 $\mathrm{N} 21$. klärt werden, dass man entweder befürchtet, mit der Anerkennung eines Lügerechts die Lüge auch moralisch zu billigen oder Beschuldigte zur Lüge zu motivieren. Weder das eine noch das andere hat die Sorge der Strafbehörden zu sein. Zur Beurteilung der moralischen Implikationen von Lügen sind Strafbehörden nicht zuständig. Ob zur Lüge gegriffen werden soll oder nicht, ist eine ausschliesslich dem Beschuldigten und seiner Strafverteidigerin zustehende Frage der Verteidigungsstrategie. Ferner ist nicht ersichtlich, weshalb, wenn man ein Recht zur Lüge verneint, man den Beschuldigten nicht gleich als Zeuge einvernimmt. 74

26 Für ein Recht zur Lüge spricht zunächst, dass Schweigen de facto kommunikativ fatale Dimensionen entfaltet, weil es als Schuldeingeständnis interpretiert wird. Schweigen kann sogar de iure zur Anerkennung des Anklagevorwurfs führen, wenn nämlich eine Schuldvermutung besteht, welche es durch Erklärungen zu widerlegen gilt. Das gilt etwa für den Wahrheits- und Gutglaubensbeweis bei der üblen Nachrede (Art. 173 Ziff. 2 StGB)75 oder für die Vermutung, dass Vermögenswerte einer Person, die sich an einer kriminellen Organisation beteiligt hat, in der Verfügungsmacht dieser Organisation stehen (Art. 72 StGB). ${ }^{76}$

74 Pfenninger, SJZ 1957 (Fn. 13), S. 147.

75 Riklin, in Niggli/Wiprächtiger (Hrsg.), Basler Kommentar, Strafrecht II, Art. 111-392 StGB, 3. Aufl., Basel 2013, Art. 173 N 13; z.R. krit. Martin Schubarth, Kommentar zum schweizerischen Strafrecht, 3. Band, Bern 1984, Art. 173 N 63 f.

76 Martin Seelmann/Marc Thommen, in: Ackermann (Hrsg.), Kommentar Kriminelles Vermögen - kriminelle Organisationen, Band 1, Zürich 2018, Art. 72 N 71. 
Fraglich ist, woraus sich das Lügerecht ergeben soll. Eine Norm, welche dem Beschuldigten das Lügen explizit erlaubt, gibt es nicht. Auch eine Aufklärung über das Recht zur Lüge wird nach geltendem Recht nicht verlangt (Art. 158 StPO).77 Auf den ersten Blick erscheint es naheliegend, das strafprozessuale Lügerecht aus dem Nemo-tenetur-Grundsatz abzuleiten. Es wurde gezeigt, dass der reformierte Strafprozess darauf verzichtet, den Beschuldigten verpflichtend in die Wahrheitsfindung einzubinden. Er hat ein durch nationales (Art. 113 StPO) und internationales (Art. 14 Ziff. 3 lit. g UNOPakt II; Art. 6 Abs. 1 und 2 EMRK) Recht abgesichertes Schweigerecht. Nach herrschender Lehre und Rechtsprechung trifft ihn keine Wahrheitspflicht. Zutreffenderweise wurde jedoch hervorgehoben, dass sich aus dem Schweigerecht keine Berechtigung zur Lüge ableiten lässt. ${ }^{8}$ Aus dem Recht nichts zu sagen, ergibt sich kein Recht etwas (Wahres oder Unwahres) zu sagen. Weder ein Recht zur Lüge noch ein Recht zur Leugnung lassen sich deshalb aus dem Nemotenetur-Grundsatz ableiten.

Dass der Beschuldigte lügen darf, ergibt sich vielmehr aus seinem Anspruch auf rechtliches Gehör und aus seiner Mei-

77 Eine solche Aufklärung postulieren überzeugend Urs Rudolf/Luzia Vetterli, Erste Erfahrungen mit der neuen StPO - am Beispiel des Kantons Luzern, forumpoenale 2011, Heft 2, S. 103 ff., 108 ( Sie haben das Recht, die Aussage oder Mitwirkung zu verweigern. Falls Sie sich entscheiden auszusagen, können die Aussagen gegen Sie verwendet werden. Sie müssen nicht die Wahrheit sagen. Sie dürfen jedoch nicht jemanden falsch beschuldigen...»).

78 Gruber (Fn. 3), S. 114 und 264 ff.; Thomas Hansjakob, Kostenarten, Kostenträger und Kostenhöhe im Strafprozess (am Beispiel des Kantons St. Gallen), Diss. St. Gallen 1988, S. 258; Schlauri (Fn. 8), S. 333. nungsäusserungsfreiheit. Das Recht im Strafverfahren Stellung zu beziehen, geht hervor aus dem rechtlichen Gehör (Art. 29 Abs. 2 BV) und aus der Garantie eines fairen Verfahrens (Art. 6 Ziff. 1 EMRK).79 Nach ständiger Rechtsprechung sind die Behörden zwar nicht verpflichtet, jegliche Parteiäusserungen im Verfahren zu berücksichtigen, der Grundsatz des rechtlichen Gehörs verlangt jedoch als Mindestvoraussetzung, dass die «Behörde die Vorbringen des Betroffenen tatsächlich hört»80. Die Behörde muss sich somit auch die Lügen des Beschuldigten anhören. Auch unwahre Äusserungen des Beschuldigten fallen somit in den Schutzbereich des rechtlichen Gehörs. ${ }^{81}$

29 Im Gegensatz zu den Freiheitsrechten, welche nach Art. 36 BV eingeschränkt werden können, gilt das rechtliche Gehör als Verfahrensgrundrecht grundsätzlich uneingeschränkt. ${ }^{82}$ Dass auch unwahre Äusserungen vom rechtlichen Gehör umfasst sind, folgt einerseits daraus, dass bis zum Verfahrensabschluss schon systembedingt gar nicht feststehen kann, ob etwa die Äusserung «ich bin unschuldig» eine Lüge ist, oder nicht. Selbst wenn sich einzelne Aussagen bereits vor Verfahrensabschluss als Lügen entlarven lassen, z.B. die durch Handy-Ortung und

79 Stefan Trechsel/Sarah J. Summers, Human Rights in Criminal Proceedings, Oxford 2005, S. 89.

8o BGE 135 III 670, E. 3.3.1.

81 Überzeugend Gruber (Fn. 3), S. 209.

82 Gerold Steinemann, in Ehrenzeller/Schindler/ Schweizer/Vallender (Hrsg.), Die Schweizerische Bundesverfassung, St. Galler Kommentar, 3. Aufl., Zürich 2014 (fortan zit.: SGK BV3Bearbeiter), Art. 29 N 8; diff. Giovanni Biaggini, BV Kommentar, Bundesverfassung der Schweizerischen Eidgenossenschaft, mit Auszügen aus der EMRK, den UNO-Pakten sowie dem BGG, Zürich 2007, Art. $36 \mathrm{~N} 4$. 
Videoaufnahmen widerlegte Behauptung zur Tatzeit im Ausland gewesen zu sein, kann dem Beschuldigten nicht untersagt werden, weiterhin an diesem vermeintlichen Alibi festzuhalten. Daran ändert auch Art. 139 StPO nichts, wonach die prozessuale «Wahrheitsfindung» (Abs. 1) insoweit eingeschränkt werden kann als über unerhebliche, offenkundige oder bereits rechtsgenügend erwiesene Tatsachen kein Beweis geführt wird (Abs. 2). Gestützt auf diese Bestimmung könnte zwar ein Beweisantrag des Beschuldigten, einen Entlastungszeugen vorzuladen, welcher sein Alibi stützt, abgelehnt, nicht aber dem Beschuldigten das Wort verboten werden. Vielmehr muss er im Strafverfahren zwingend angehört werden.

Das Recht auf Anhörung ist ein "prozessuales Urrecht» des Beschuldigten, welches unter anderem verhindern soll, dass er zum reinen Objekt des Verfahrens degradiert wird. ${ }^{83}$ Die Strafbehörden müssen der beschuldigten Person Gelegenheit geben, «sich zu diesen Straftaten umfassend zu äussern» (Art. 157 Abs. 2 StPO). Nach Art. 341 Abs. 3 StPO ist die beschuldigte Person in der Hauptverhandlung eingehend zu ihrer Person, zur Anklage und zu den Ergebnissen des Vorverfahrens zu befragen. Keine dieser Normen, welche dem Beschuldigten das Recht geben, sich zu äussern, schränkt das Äusserungsrecht inhaltlich auf «wahre» Äusserungen ein. Eine solche Einschränkung ergibt sich auch nicht aus dem Rechtsmissbrauchsverbot. Erstens richtet sich Art. 3 Abs. 2 lit. b StPO nach seinem Wortlaut nur an die Strafbehörden. Doch selbst wenn man mit dem

83 Urteil des Bundesverfassungsgerichts 107, 395, $407 \mathrm{f}$.
Bundesgericht zweitens ein allgemeines, auch für den Beschuldigten geltendes Rechtsmissbrauchsverbot bejahte, hätte dies keinen Einfluss auf sein Lügerecht. ${ }^{84}$ Ein Beschuldigter, der lügt, missbraucht nicht das Institut des rechtlichen Gehörs, ${ }^{85}$ sondern nimmt dieses Recht vielmehr für denjenigen Zweck in Anspruch, für den es geschaffen wurde, nämlich seine Position in das Strafverfahren einzubringen. Der Beschuldigte hat somit ein Recht, Lügen im Strafverfahren zu äussern. Damit ist freilich noch nicht gesagt, dass Lügen keine Konsequenzen haben können.

31 Das Recht des Beschuldigten, sich zu äussern, ergibt sich möglicherweise auch aus seiner Meinungsäusserungsfreiheit. Nach Art. 16 Abs. 2 BV hat jede Person das Recht, ihre Meinung ungehindert zu äussern und $\mathrm{zu}$ verbreiten. Meinungen umfassen dabei «die Gesamtheit der Mitteilungen menschlichen Denkens» ${ }^{86}$. Auf den Inhalt einer Meinungsäusserung kommt es grundsätzlich nicht an. ${ }^{87} \mathrm{Ge}-$ schützt sind auch unhaltbare und offensichtlich falsche Äusserungen. ${ }^{88}$ Auch die

84 Kritik dazu BSK StPO'2-Thommen, Art. 3 N 78.

85 Zum sog. Institutsmissbrauch als Hauptanwendungsfall des Rechtsmissbrauchs BGE 131 II 265 E. 4.2. m.w.H.

86 BGE 127 I 145, E. 4b; s.a. Urteil des EGMR 5493/72 vom 7. Dezember 1976 (Handyside $v$. The United Kingdom), Ziff. 49.

87 BGE 138 I 274.

88 Hertig, in Waldmann/Belser/Epiney (Hrsg.), Basler Kommentar, Schweizerische Bundesverfassung (BV), Basel 2015 (fortan zit.: BSK BVBearbeiter), Art. $16 \mathrm{~N}$ 9; Schmalenbach, (Fn. 10), S. 752; ob Lügen unter den Schutzbereich der Meinungsäusserungsfreiheit fallen, ist insb. in Deutschland allerdings umstritten, für den dortigen Streitstand vgl. Gruber (Fn. 3), S. 85 ff. und Schmalenbach (Fn. 10), S. 750. Das dürfte wohl auch für die Schweiz zutreffen vgl. SGK BV3Kley/Tophinke, Art. 16 N 5, wonach der Begriff der Meinung sich auf Tatsachendarstellungen beziehe m.H.a. BGE 113 Ia 309, E. 5a. 
Meinungsäusserungsfreiheit gewährt dem Beschuldigten somit das Recht, in Strafverfahren Lügen zu äussern. Selbstverständlich gilt dieses Recht nicht uneingeschränkt. Freiheitsrechte unterliegen nach Massgabe von Art. 36 BV der Einschränkung. ${ }^{89}$ Ein Recht zur Lüge wird oft mit dem Argument angezweifelt, dass sich der Beschuldigte mit unwahren Aussagen der Falschanschuldigung oder der Irrführung der Rechtspflege strafbar machen könne. ${ }^{\circ 0}$ Gemäss dem schweizerischen Bundesgericht könne «ein allfälliges Recht zu lügen nur innerhalb der Grenzen des Strafrechts Geltung erlangen».91 Diese Einwände sprechen indes nicht gegen das Lügerecht als solches, sondern betreffen lediglich dessen Umfang. Dass ein Recht Einschränkungen unterliegt, ist kein zureichender Grund, ihm den Charakter als Recht abzusprechen. So käme auch niemand auf die Idee, ein Recht auf Versammlungsfreiheit (Art. 22 BV) zu verneinen, nur weil diese polizeilichen oder strafrechtlichen 92 Einschränkungen unterliegt.

\section{Zusammenfassung}

32 Die Eingangsfrage ist zu bejahen: Der Beschuldigte darf im Strafverfahren lügen. Damit ist freilich noch nicht gesagt, dass seine Lügen keine faktischen (z.B. U-Haft) oder rechtlichen Folgen (z.B. strafbare Falschanschuldigung) haben können. Das grundrechtlich abgesicherte Recht zur Lüge kann in verschiedener Hinsicht durch strafprozessuale oder kernstrafrechtliche Normen einge-

89 Biaggini (Fn. 82), Art. $36 \mathrm{~N} 9$ ff.

90 BSK StPO2-Häring, Vor Art. 142-146 N 13; Schmid (Fn. 72) 2004, N 272.

91 Urteil des Bundesgerichts 6B_157/2016 vom 8. August 2016, E. 2.4.2.

92 Vgl. etwa Art. 260 StGB Landfriedensbruch. schränkt werden.93 Diese strafprozessualen Lügenfolgen und materiell-strafrechtlichen Lügenstrafen bedürfen einer eingehenden Betrachtung, für die der Raum hier allerdings fehlt. Dass der Beschuldigte lügen darf, bedeutet zusammenfassend nicht, dass er lügen soll. Tut er es aber, dann muss sich das Gericht auch «totalen Quatsch» anhören. Darin liegt die Essenz des Lügerechts.
$93 \overline{\text { Gleich Gruber (Fn. 3), S. 209; Schmalenbach }}$ (Fn. 10), S. 752. 